\title{
Exposição ocupacional aos agrotóxicos da classe dos fungicidas em uma população de viticultores
}

\author{
Occupational exposure to pesticides from fungicide class on a vineyard farm population \\ Exposición ocupacional a plaguicidas de la clase fungicida en una población de viticultores
}

Recebido: 11/03/2021 | Revisado: 18/03/2021 | Aceito: 20/03/2021 | Publicado: 27/03/2021

Renata Sano Lini

ORCID: https://orcid.org/0000-0002-6161-2225 Universidade Estadual de Maringá, Brasil

E-mail: renatalini23@gmail.com

Nadya Garcia de Oliveira

ORCID: https://orcid.org/0000-0002-1753-8996 Universidade Estadual de Maringá, Brasil

E-mail: nadyagarcia9@gmail.com

Bruna Giovana Nery

ORCID: https://orcid.org/0000-0002-8360-2239 Universidade Estadual de Maringá, Brasil E-mail: bruna_nery@hotmail.com

Raul Gomes Aguera

ORCID: https://orcid.org/0000-0002-6090-8585 Universidade Estadual de Maringá, Brasil E-mail: raul1994_gomes@hotmail.com

Laís Fernanda Ferreira da Silva

ORCID: https://orcid.org/0000-0001-5112-268X

Universidade Estadual de Maringá, Brasil E-mail: la_isfernanda@hotmail.com

Samuel Botião Nerilo

ORCID: https://orcid.org/0000-0003-2527-8503 Universidade Estadual de Maringá, Brasil E-mail: samuelnerilo@gmail.com

Sílvia Capelari

ORCID: https://orcid.org/0000-0001-6552-1526 Universidade Estadual de Maringá, Brasil E-mail: scapelari@gmail.com

Magda Lúcia Félix de Oliveira

ORCID: https://orcid.org/0000-0003-4095-9382 Universidade Estadual de Maringá, Brasil E-mail: mlfoliveira@uem.br

Simone Aparecida Galerani Mossini ORCID: https://orcid.org/0000-0001-9535-0983 Universidade Estadual de Maringá, Brasil E-mail: sagmossini@uem.br

\section{Resumo}

A exposição crônica aos agrotóxicos pode levar ao desenvolvimento de doenças, e a escassez de informações sobre manifestações clínicas na exposição a longo prazo é uma preocupação atual. Este trabalho teve como objetivo analisar o uso crônico de agrotóxicos da classe dos fungicidas por viticultores de um município do Paraná, região sul do Brasil. Foram entrevistados 192 agricultores familiares, que residiam em 66 propriedades, com instrumentos desenvolvidos segundo o protocolo de avaliação das intoxicações crônicas por agrotóxicos da Secretaria de Estado da Saúde do Paraná. Entre os participantes, 45,8\% trabalhava há mais de 20 anos na viticultura, utilizando continuamente os fungicidas e apenas $6,8 \%$ relatou uso de todos os equipamentos de proteção recomendados para a aplicação dos agrotóxicos. Foram relatados sintomas como irritação cutânea pruriginosa $(20,5 \%)$ e tremores $(12,1 \%)$ que podem estar associados a exposição crônica aos fungicidas ditiocarbamatos. A população agricultora familiar carece de assistência à saúde, fato relatado também pela população estudada, evidenciando falhas no serviço de atenção e a necessidade de novas estratégias e tecnologias de acesso a esse grupo.

Palavras-chave: Exposição ocupacional; Agroquímicos; Fungicidas industriais; Saúde pública.

\section{Abstract}

Chronic exposure to pesticides can lead to disease and the scarcity of information on clinical manifestations and long term poisoning is a current concern. The aim of this work was to analyze the chronic use of pesticides of the fungicide 
class by a population of wine grower workers from a southern region of Brazil. A hundred and ninety-two family farmers were interviewed with instruments developed according to the protocol for the evaluation of chronic poisoning by pesticides of the Paraná State Department of Health. Among the participants, $45.8 \%$ worked in viticulture for over 20 years, continuously using fungicides, and only $6.8 \%$ used all protective equipment recommended for pesticide application. There were reported symptoms such as itchy skin irritation $(20.5 \%)$ and tremors $(12.1 \%)$ that may be associated with the chronic exposure to dithiocarbamate fungicides. The population of family farmers lacks health care, a fact also reported by the population studied. This highlights failures in the care service and the need for new strategies and technologies to access this group.

Keywords: Occupational exposure; Agrochemicals; Fungicides, industrial; Public health.

\section{Resumen}

La exposición crónica a plaguicidas puede conducir al desarrollo de enfermedades, y la escasez de información sobre las manifestaciones clínicas en la exposición crónica a fungicidas es una preocupación actual. Este estudio tuvo como objetivo analizar el uso crónico de plaguicidas de la clase fungicida por viticultores en un municipio de Paraná, sur de Brasil. Se entrevistaron 192 agricultores familiares, que vivían en 66 propiedades, con instrumentos desarrollados de acuerdo con el protocolo para la evaluación de intoxicaciones crónicas por plaguicidas del Departamento de Salud del Estado de Paraná. Entre los participantes, el 45,8\% había trabajado en viticultura durante más de 20 años, utilizando fungicidas de forma continúa y solo el 6,8\% informó utilizar todos los equipos de protección recomendados para la aplicación de plaguicidas. Algunos informaron síntomas como irritación cutánea con picazón $(20,5 \%)$ y temblores $(12,1 \%)$ que pueden estar asociados con la exposición crónica la clase de fungicidas de ditiocarbamato. La población de agricultores familiares carece de atención médica, hecho también reportado por la población estudiada. Esto pone de relieve las fallas en el servicio de atención y la necesidad de nuevas estrategias.

Palabras clave: Exposición ocupacional; Agroquímicos; Fungicidas industriales; Salud pública.

\section{Introdução}

Agrotóxicos são amplamente utilizados no combate de pragas e doenças para elevar a produtividade agrícola e o Brasil ocupa posição de destaque como consumidor de agrotóxicos no mundo. Segundo o boletim anual do Instituto Brasileiro do Meio Ambiente e dos Recursos Naturais Renováveis (2018), mais de 549.000 toneladas de insumo ativo foram consumidos no país em 2018.

O uso excessivo destes produtos acarreta prejuízos à saúde dos agricultores que os manipulam, muitas vezes, sem a proteção adequada (Furlong, et al., 2015; García-García, et al., 2016). No ano de 2019, foram registrados 5.700 casos de intoxicações agudas por agrotóxicos agrícolas, e a maior parte dos eventos se concentraram nas regiões Sul e Sudeste do país (Departamento de Informática do Sistema Único de Saúde, 2019). Um indicador que geralmente apresenta subnotificação nas estatísticas dos sistemas de informação de saúde do Brasil, é a prevalência de intoxicação crônica por agrotóxicos (Secretaria de Estado da Saúde do Paraná, 2013). A literatura aponta a relação da exposição crônica ao aparecimento de doenças como Parkinson (Gunnarsson \& Bodin, 2019; Mostafalou \& Abdollahi, 2013; Van der Mark, et al., 2012; Yan, et al., 2018), distúrbios da tireoide (Mostafalou \& Abdollahi, 2013; Medda, et al., 2017; Nascimento, et al., 2018) e problemas respiratórios (Mostafalou \& Abdollahi, 2013; Buralli, et al., 2018; Ye, et al., 2017).

Apesar do grande número de estudos envolvendo o aparecimento de doenças e o uso prolongado de agrotóxicos, é difícil encontrar na literatura pesquisas relacionadas às manifestações clínicas em exposição crônica (Lopes \& de Albuquerque, 2018). De acordo com o Dossiê elaborado pela Associação Brasileira de Saúde Coletiva (ABRASCO) (Carneiro, 2015), a exposição prolongada à classe dos fungicidas pode causar alterações psicomotoras (tremores), respiratórias (tosse persistente), e dermatológicas (irritação cutânea pruriginosa). A presença destes sinais geralmente antecede o aparecimento de doenças de maior gravidade, e torna importante monitorar a saúde de trabalhadores rurais expostos por vários anos.

São poucos estudos que relacionam o uso prolongado de fungicidas com o aparecimento de sintomas clínicos. Além disso, as pesquisas envolvendo esta classe de agrotóxicos geralmente se limitam a apenas um produto da classe, como o estudo de Medda, et al. (2017) que percebeu relação entre o uso de mancozebe (um fungicida) e efeitos na glândula tireoide de agricultores da Itália. 
A cultura da uva, em locais de clima quente e úmido, exige uso intensivo de agrotóxicos principalmente da classe dos fungicidas. Os agricultores que trabalham com essa cultura, quando não utilizam os equipamentos de proteção adequados, estão diariamente expostos aos fungicidas (Mossini, et al., 2017; Rocha, et al., 2015). Neste contexto, a presente proposta foi analisar o uso crônico de fungicidas por viticultores de uma região no noroeste do Paraná.

\section{Materiais e Métodos}

O estudo seguiu as recomendações metodológicas descritas por Pereira et al. (2018), que traz orientações para melhorar a qualidade de pesquisas científicas. Trata-se de um estudo observacional transversal, na modalidade de estudo de caso, com abordagem qualitativa e quantitativa, realizado em propriedades agrícolas e com agricultor familiar envolvido na cultura da uva, em Marialva - PR, abrangendo a tríade observação-entrevista-coleta de material biológico.

O estado do Paraná é um grande produtor de uva, e dentre os municípios produtores, Marialva é um território vitivinícola com produção de uva convencional, consolidado em nível estadual e nacional e conhecido como a Capital da Uva Fina. O município possui, aproximadamente 35.000 habitantes e localiza-se na região noroeste do Paraná, e apesar de grande produção agrícola, a viticultura realizada no município possui particularidades como a pequena propriedade, a mecanização de baixa tecnologia e principalmente a utilização de mão de obra familiar (Zeneratti, 2019).

O protocolo de avaliação das intoxicações crônicas por agrotóxicos foi utilizado como referencial para contextualização, elaboração do instrumento utilizado para entrevista e discussão dos dados. Este material foi criado como parte de ações da Secretaria de Estado da Saúde do Paraná para enfrentar a problemática do uso de agrotóxicos, de forma que auxilia os profissionais da área da saúde a direcionar o atendimento, o diagnóstico e a vigilância de casos de intoxicação crônica (Secretaria de Estado da Saúde do Paraná, 2013).

A seleção dos participantes foi realizada de forma intencional, em parceria com técnicos do Instituto de Desenvolvimento Rural do Paraná (IDR-Paraná) de Marialva, que forneceu a listagem de produtores de uva cadastrados em seu banco de dados.

Dos 76 produtores cadastrados, 48 aderiram à investigação. No entanto, durante o período de deslocamento de membros da equipe de pesquisa de campo nas propriedades rurais dos trabalhadores investigados, para inspeção das condições ambientais e contato inicial com as famílias, 18 viticultores que não estavam cadastrados no banco de dados do IDR-Paraná, solicitaram inclusão na pesquisa e foram incluídos, totalizando 66 participantes. Consignados os trabalhadores e familiares que conviviam nas propriedades, os participantes no estudo foram 192 pessoas.

A coleta de dados foi realizada durante o ano de 2017, por meio de entrevistas e com o mínimo de duas visitas técnicas às propriedades rurais, com observação não participante, agendada previamente com o produtor. Para a entrevista foram desenvolvidos dois instrumentos, um que continha informações sociodemográficas, presença de doenças crônicas não transmissíveis autorreferidas, hábitos de vida (tabagismo e consumo de álcool), forma e tempo de contato com agrotóxicos, e uso de equipamento de proteção individual.

O instrumento 2 envolvia informações relacionadas à propriedade de produção familiar como o percentual e a indicação do uso de agrotóxicos, forma de aplicação/ armazenamento/ descarte das embalagens dos agrotóxicos, além de políticas sociais percebidas pela família. Todos os resultados autorreferidos foram compilados para planilhas do software Excel $^{\circledR}$, onde foi realizada análise descritiva simples.

Para o levantamento de informações sobre o uso de agrotóxicos, foi utilizada a listagem de produtos, com denominação comercial, indicados pelo IDR-Paraná de Marialva aos viticultores, e no momento da entrevista foi perguntado se os mesmos utilizavam estes produtos na propriedade rural. Para o detalhamento do grupo químico e classificação toxicológica dos agrotóxicos foi utilizado o site da Agência de Defesa Agropecuária do Paraná (2019a). 
Além da entrevista, também foi realizada a análise da atividade enzimática da colinesterase em sangue total, único parâmetro reconhecido pela legislação brasileira para o monitoramento biológico da exposição ocupacional a agrotóxicos (Ministério do Trabalho e Emprego, 1978). Para esta análise, o sangue foi coletado em tubos de coleta a vácuo de 6,0 mL heparinizados da marca VACUETTE®. Após, as amostras foram enviadas ao Laboratório de Toxicologia da Universidade Estadual de Maringá, sob refrigeração, onde foram processadas e posteriormente submetidas à análise. Quando necessário, as amostras foram congeladas a $-20{ }^{\circ} \mathrm{C}$. A determinação da atividade enzimática foi realizada seguindo o método descrito em Ellman, et al. (1961) modificado por Harlin \& Ross (1990). A absorbância da reação foi medida em 412nm em espectrofotômetro UV-visível (Shimadzu UV-1601PC).

O estudo foi aprovado pelo Comitê Permanente de Ética em Pesquisa Envolvendo Seres Humanos (COPEP) da Universidade Estadual de Maringá, parecer nº 2.068.991.

\section{Resultados}

A Tabela 1 apresenta características dos 192 participantes. A maioria eram homens, com média de idade de 47,2 $\pm 13,1$ anos, sendo a faixa etária entre 46 e 60 anos predominante. A maioria possuía ensino fundamental, e poucos possuíam ensino superior; e acima da metade dos viticultores eram "autônomos", caracterizados pela ausência de contribuição com o Instituto Nacional do Seguro Social - INSS. As famílias eram constituídas, em média, por 2,9ะ1,2 pessoas com renda familiar média de R \$2.240,60 (salário mínimo da época R\$937,00) (Brasil, 2016). 
Tabela 1: Variáveis sociodemográficas de agricultores familiares $(n=192)$ ocupacionalmente expostos a fungicidas. MarialvaPR, 2017.

\begin{tabular}{|c|c|c|}
\hline Variáveis & n $(\%)$ & Média \pm DP \\
\hline Idade (anos) & & $47,2 \pm 13,1$ \\
\hline $18-30$ & $23(12,0)$ & \\
\hline $31-45$ & $66(34,4)$ & \\
\hline $46-60$ & $69(35,9)$ & \\
\hline$>60$ & $34(17,7)$ & \\
\hline \multicolumn{3}{|l|}{ Sexo } \\
\hline Masculino & $108(56,3)$ & \\
\hline Feminino & $84(43,8)$ & \\
\hline \multicolumn{3}{|l|}{ Escolaridade } \\
\hline Ensino Fundamental C e I & $104(54,2)$ & \\
\hline Ensino Médio C e I & $75(39,1)$ & \\
\hline Ensino Superior C e I & $4(2,1)$ & \\
\hline Não frequentou & $4(2,1)$ & \\
\hline NR/NS & $5(2,6)$ & \\
\hline \multicolumn{3}{|c|}{ Situação no mercado de trabalho } \\
\hline Aposentado & $10(5,2)$ & \\
\hline Autônomo & $139(72,4)$ & \\
\hline Empregado não registrado & $25(13,0)$ & \\
\hline Empregado registrado & $2(1,0)$ & \\
\hline $\mathrm{NR} / \mathrm{NS}$ & $16(8,3)$ & \\
\hline \multicolumn{3}{|l|}{ Contribuição do INSS } \\
\hline Sim & $36(18,8)$ & \\
\hline Não & $150(78,1)$ & \\
\hline NR/NS & $6(3,1)$ & \\
\hline Pessoas na família & & $2,9 \pm 1,2$ \\
\hline 1 a 5 & $166(86,5)$ & \\
\hline Mais que 5 & $4(2,1)$ & \\
\hline NR/NS & $22(11,5)$ & \\
\hline Renda familiar (reais) & & $2.240,60 \pm 1.153,80$ \\
\hline$<1.000$ & $18(9,4)$ & \\
\hline 1.000 a 1.999 & $61(31,8)$ & \\
\hline 2.000 a 2.999 & $50(26,0)$ & \\
\hline$\geq 3.000$ & $39(20,3)$ & \\
\hline NR/NS & $24(12,5)$ & \\
\hline
\end{tabular}

n: Quantidade de pessoas; \%: Percentual; DP: Desvio padrão; C: Completo; I: Incompleto; NR: Não respondeu; NS: Não sabe. Fonte: Autores.

Os agrotóxicos utilizados pelos viticultores com a respectiva porcentagem de uso, estão listados na Tabela 2, segundo composto ativo, grupo químico e classificação toxicológica segundo a Agência Nacional de Vigilância Sanitária (2015). Todos pertencem à classe dos fungicidas, com exceção da cianamida que é um regulador de crescimento. Todos os fungicidas apresentaram alto percentual de uso, sendo o grupo químico dos ditiocarbamatos o mais citado. É importante destacar que as substâncias listadas se referem àquelas utilizadas no momento da entrevista (exposição recente), porém os entrevistados relataram que estes agrotóxicos são utilizados por eles para a cultura da uva há muitos anos. 
Tabela 2: Utilização de fungicidas recomendados para a cultura de uva. Propriedades de agricultores familiares ( $\mathrm{n}=66)$, Marialva-PR, 2017.

\begin{tabular}{cccc}
\hline Composto ativo & Grupo químico & $\begin{array}{c}\text { Classificação } \\
\text { Toxicológica }\end{array}$ & Percentual \\
\hline Tiofanato metilico & Benzimidazol & $\mathrm{V}$ & 95,8 \\
Propiconazol e Difenoconazol & Triazol & $\mathrm{V}$ & 95,3 \\
Piraclostrobina e Metiram & Estrobilurina e Ditiocarbamato & $\mathrm{IV}$ & 95,3 \\
Mancozebe & Ditiocarbamato & $\mathrm{V}$ & 93,7 \\
Oxicloreto de cobre & Inorgânico & $\mathrm{V}$ & 92,1 \\
Benalaxil e Mancozeb & Acilalaninato e Ditiocarbamato & $\mathrm{V}$ & 88,9 \\
Cimoxanil e Mancozebe & Acetamida e Ditiocarbamato & $\mathrm{V}$ & 88,4 \\
Fosetyl & Fosfonato & Não Classificado & 83,7 \\
Fenamidona & Imidazolinona & $\mathrm{V}$ & 77,9 \\
Metalaxil-M e Clorotanil & Acilalaninato e Isoftalonitrila & III & 72,1 \\
Folpete & Dicarboximida. & $\mathrm{V}$ & 70,0 \\
Tebuconazol & Triazol & $\mathrm{V}$ & 66,3 \\
Iprodiona & Dicarboximida & $\mathrm{V}$ & 65,3 \\
Mancozebe & Ditiocarbamatos & $\mathrm{V}$ & 59,5 \\
Hidróxido de cobre & Inorgânico & $\mathrm{V}$ & 46,8 \\
Azoxistrobina e Difenoconazol & Estrobilurina e triazol & $\mathrm{V}$ & 36,3 \\
Captana & Dicarboximida & IV & 26,3 \\
\hline
\end{tabular}

III: Moderadamente tóxico; IV: Pouco tóxico; V: Improvável de causar dano agudo; Não classificado: Produto não classificado como tóxico. Fonte: Autores.

A principal forma de aplicação dos agrotóxicos era por mecanização de baixa tecnologia, pulverizador mecanizado (trator), em $61,7 \%(n=41)$ das propriedades. Com relação à orientação para uso de agrotóxicos, 75,0\% (n=49) dos produtores disseram que seguiam a indicação de um engenheiro agrônomo na hora da compra. 93,2\% (n=62) dos viticultores relataram utilizar o receituário agronômico para a compra dos agrotóxicos e também seguiam as instruções contidas no receituário. Porém, apenas 13,6\% (n=9) dos participantes armazenavam os agrotóxicos em local seguro - armário trancado específico para produtos químicos em construção distante da casa onde residem. Em grande parte das propriedades (n=52; 78,3\%), os agrotóxicos eram armazenados, juntamente com outros produtos químicos, em local externo à residência, porém não trancado. Os participantes disseram que as embalagens vazias, em 81,7\% ( $n=54)$ dos casos, eram recolhidas no Centro de Triagem e Reciclagem de Agrotóxicos de Marialva.

Grande parte dos participantes trabalhava há mais de 20 anos na agricultura (n=88; 45,8\%), e eram expostos cotidianamente aos agrotóxicos por meio do trabalho $(n=174 ; 90,6 \%)$. Poucos $(n=13 ; 6,8 \%)$ relataram fazer o uso do Equipamento de Proteção Individual (EPI) recomendado de forma integral. A Tabela 3 demonstra o perfil do uso de EPI por esta população. 
Tabela 3: Perfil do uso de EPI por população de agricultores familiares ( $n=192)$ exposta aos fungicidas. Marialva-PR,2017.

\begin{tabular}{lc}
\hline EPI & Percentual \\
\hline Usam todos os recomendados & 6,8 \\
Não usam & 38,5 \\
Usam parcialmente & \\
Luvas & 54,7 \\
Botas & 46,4 \\
Máscara & 38,5 \\
Macacão & 25,5 \\
Viseira & 12,0 \\
\hline
\end{tabular}

EPI: Equipamento de Proteção Individual. Fonte: Autores.

Com relação ao histórico de agravos e doenças, grande parte dos viticultores relatou hipertensão arterial sistêmica ( $\mathrm{n}=61$; $31,8 \%)$, transtorno mental menor/ansiedade $(n=52 ; 27,1 \%)$ e seis deles relataram neoplasias $(3,0 \%)$, com realização de cirurgia reparadora de mama, pele, próstata, útero, linfoma (virilha) e intestino.

Os viticultores referiram, no momento da entrevista, outros problemas de saúde crônicos (n=78; 40,6\%) e estavam fazendo uso de medicamentos, prescritos ou por automedicação $(\mathrm{n}=89 ; 46,4 \%) .9,9 \%(\mathrm{n}=19)$ eram tabagistas, e 50,0\% (n=96) usuários de bebida alcoólica, embora parte deles não informou o uso nocivo.

Em relação ao atendimento em saúde, grande parte utilizava o Sistema Único de Saúde (SUS), em hospitais públicos $(\mathrm{n}=29 ; 43,3 \%)$, ou em unidades básicas de saúde $(\mathrm{n}=17 ; 25,0 \%)$. Quanto à saúde familiar, apenas 3,3\% (n=2) dos agricultores proprietários informaram que pelo menos um membro da família "adoeceu" em 2016, e 10,0\% (n=7) declararam que algum familiar necessitou de internamento neste mesmo ano. Em 8,3\% $(n=6)$ das propriedades houve morte de pelo menos um familiar também em 2016.

Com relação à percepção dos viticultores quanto às políticas sociais existentes na região envolvendo a agricultura, mais da metade $(n=35 ; 52,5 \%)$ sentem-se desamparados, e 59,0\% (n=39) relataram sentir-se abandonados. A saúde foi o serviço público em que quase a metade dos produtores $(n=3045,9 \%)$ disseram ser pior atendidos. Grande parte $(n=55 ; 83,6 \%)$ relatou não receber visita domiciliar periódica de agentes comunitários de saúde, e 86,9\% (n=57) não tinham assistência de equipes de Saúde da Família.

Alguns participantes $(n=22 ; 11,5 \%)$ relataram já ter se intoxicado de forma aguda com os agrotóxicos utilizados para a cultura da uva, sendo que em 3,1\% (n=6) dos casos, a intoxicação ocorreu mais de uma vez. Dos trabalhadores que se intoxicaram, 40,9\% (n=9) não procurou qualquer atendimento médico, e daqueles que foram assistidos em serviços de saúde (n=13), em 27,3\% (n=4) casos aconteceu em hospital público do município.

As queixas de saúde relatadas pelos viticultores e que poderiam estar associadas à exposição crônica a fungicidas, foram organizadas na Figura 1. A alteração dermatológica/ irritação cutânea pruriginosa foi a queixa mais autorreferida, seguido de alterações psicomotoras/tremores, e sintomas associados ao sistema respiratório. Os agricultores familiares referiram crença de que doenças como câncer e hipotireoidismo teriam relação com uso de agrotóxicos. 
Figura 1: Doenças e sintomas citados pelos viticultores que podem estar relacionados à intoxicação crônica por ditiocarbamatos (Secretaria de Estado da Saúde do Paraná, 2013).

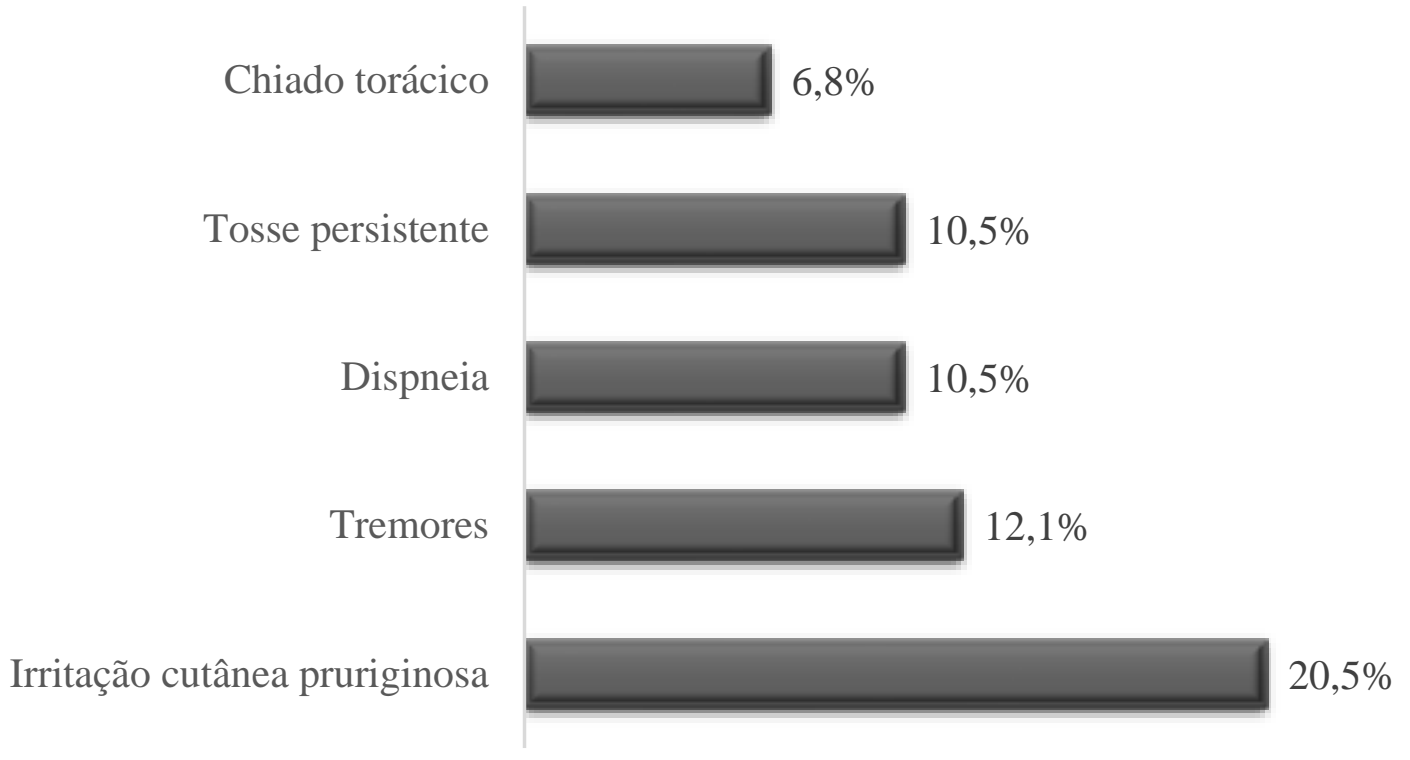

Fonte: Autores.

Com relação a atividade da enzima colinesterase em sangue total dos viticultores, a média encontrada para os homens foi de $4.4 \pm 0.7 \mu \mathrm{mol} / \mathrm{ml} / \mathrm{min}$ e para as mulheres $4.1 \pm 0.5 \mu \mathrm{mol} / \mathrm{ml} / \mathrm{min}$. Como os viticultores não ficam afastados da aplicação de agrotóxicos por pelo menos 30 dias, não foi possível realizar a porcentagem de inibição da enzima como estabelecido na legislação brasileira.

\section{Discussão}

Viabilizar o conhecimento sobre uso de agrotóxicos é uma etapa fundamental para regular o uso destes produtos entre os agricultores, o embasamento científico desconstrói algumas crenças e auxilia a mudança de atitudes dos agricultores que são resistentes a alterações na rotina da prática da agricultura convencional.

Assim como observado em outros estudos (Buralli, et al., 2018; Raksanam, et al., 2014; Viana, et al., 2017) a maioria dos trabalhadores envolvidos com o cultivo da uva na região estudada apresentava baixo nível de escolaridade. Estudos, como o de Raksanam, et al. (2014), inferem que o baixo nível de escolaridade atrapalha no entendimento das instruções de segurança contida nas embalagens. Apesar de uma porcentagem significativa de viticultores possuir baixa escolaridade, este fato não foi relacionado com o entendimento dos viticultores acerca do conteúdo das embalagens dos agrotóxicos, porém, pode estar associado com a falta de cuidado com a manipulação dos produtos.

Antes da reclassificação toxicológica realizada pela Agência Nacional de Vigilância Sanitária em 2017, a maioria dos produtos utilizados pelos viticultores eram classificados como "extremamente tóxico", mas após a reclassificação, a categoria V, que se refere aos produtos que são improváveis de causar dano agudo, prevaleceu (Tabela 2) (Agência Nacional de Vigilância Sanitária, 2015). A cianamida apresentou maior percentual de uso entre os agricultores. Este regulador de crescimento, utilizado para quebra do período de dormência da planta, é extremamente tóxico sendo que a intoxicação aguda pode causar efeitos severos (Agência de Defesa Agropecuária do Paraná, 2019b). Dentre os fungicidas, a classe dos ditiocarbamatos é a que se fez mais presente no uso da viticultura. Cabe ressaltar que esta classe de fungicida apresenta o metal manganês em sua formulação, sendo este um conhecido causador de problemas neurológicos (Salgado, et al., 1987). 
O fato de grande parte desta população seguir a indicação do engenheiro agrônomo e fazer uso do receituário agronômico no momento da compra do agrotóxico, demonstra que os produtores são assistidos por profissionais capacitados mesmo sendo pequenos produtores. Diferente do encontrado por Soares \& Porto (2012) que em seu estudo, também realizado no estado do Paraná, observou que pequenas propriedades do estado não tinham assistência técnica adequada.

O armazenamento dos agrotóxicos deve ser feito fora da casa e em local trancado para evitar acidentes (Alencar, 2010). Os entrevistados em sua maioria, mantinham os agrotóxicos em local fora da casa, porém não em local trancado. Quanto ao destino final das embalagens de agrotóxicos, o recomendado é que não sejam descartadas em lixo comum (Brasil, 1989). A maioria dos participantes dão o destino correto às embalagens. A aplicação do agrotóxico por pulverizador mecanizado, associado à entrega das embalagens vazias para o local correto, e a utilização do receituário agronômico por grande parte dos viticultores, revela que a população estudada possui acesso e segue as orientações técnicas fornecidas por profissionais da área.

A maioria dos viticultores se expõe aos agrotóxicos cronicamente, já que grande parte trabalha com agricultura há mais de 20 anos. Tendo em vista esse tempo prolongado de exposição, é preciso levar em conta que estes trabalhadores podem ter sido expostos a uma diversidade de agrotóxicos, e ter o conhecimento exato de todos é extremamente difícil. Outro fator preocupante é o uso do EPI por pequena parte da população, gerando um quadro de exposição crônica agravado pela falta de proteção. Apesar da utilização do EPI recomendado ser uma forma do trabalhador rural se proteger ao manusear os agrotóxicos, a falta do uso destes equipamentos também foi observada em outros estudos brasileiros que demostram como os agricultores sabem da importância do EPI, entretanto não o utilizam (Sousa, et al., 2016; Viana, et al., 2017).

A maioria dos agrotóxicos, citados por esta população, demanda o uso de macacão, botas, avental, máscara, óculos, touca árabe e luvas. Os viticultores relataram que não fazem uso de todos estes equipamentos por conta do desconforto causado, muitos mencionaram que sentem muito calor e dificuldade em se movimentar enquanto estão trabalhando com os equipamentos.

Já que a falta de adesão ao uso do EPI é um problema observado em outros estudos, é necessário pensar em outros meios de proteger o trabalhador rural. Uma alternativa para promover a segurança, seria substituir e/ou associar o uso dos agrotóxicos a métodos alternativos, como por exemplo, o controle biológico. O IDR-Paraná de Marialva tem orientado os trabalhadores rurais entrevistados a reduzir o número de princípios ativos utilizados na plantação da uva, associando métodos alternativos e evitando o uso de mais de um agrotóxico com a mesma finalidade.

Alguns agricultores relataram casos de intoxicação ocupacional aguda. Pesquisa realizada nesta mesma região do Paraná revela que as intoxicações ocupacionais geralmente ocorrem no meio agrícola e que o agrotóxico representa o agente com maior potencial letal entre os casos de internação hospitalar por intoxicação (Hungaro, et al., 2015). Felizmente os acontecimentos informados não foram letais, e menos da metade dos intoxicados procuraram atendimento médico.

Diferente da intoxicação aguda, a intoxicação crônica dificilmente é identificada e notificada. Faltam instrumentos clínicos que auxiliem o profissional de saúde a diagnosticar este tipo de intoxicação e isso leva a subnotificação destes casos (Secretaria de Estado da Saúde do Paraná, 2013). Alguns dos sintomas relatados pela população de agricultores familiar podem estar relacionados com a exposição crônica aos ditiocarbamatos, porém, são sintomas inespecíficos (Secretaria de Estado da Saúde do Paraná, 2013). Também a presença de algumas doenças relatadas pelos participantes pode estar relacionada com a atividade laboral, os casos de câncer principalmente. As revisões bibliográficas de Kim, et al. (2017) e Mostafalou \& Abdollahi (2013) mostram que doenças como asma, diabetes, Parkinson, leucemia e cânceres, podem estar associadas com a exposição crônica aos agrotóxicos.

Com relação ao biomarcador de exposição aos agrotóxicos, como não foi possível determinar o valor de atividade basal dos viticultores, foram utilizados valores de referência obtidos por Nerilo, et al. (2010) em seu estudo com populações expostas e não expostas aos inseticidas inibidores da colinesterase (valor de referência para homens: 5,6 $\mu \mathrm{mol} / \mathrm{ml} / \mathrm{min}$ a $7,0 \mu \mathrm{mol} / \mathrm{ml} / \mathrm{min}$ e para mulheres: $4,9 \mu \mathrm{mol} / \mathrm{ml} / \mathrm{min}$ a $6,3 \mu \mathrm{mol} / \mathrm{ml} / \mathrm{min}$ ). Este estudo foi escolhido por ser o único, do conhecimento dos autores, 
que apresenta valores de referência para colinesterase em sangue total para a população que reside na região de interesse. A média encontrada neste estudo, tanto para homens quanto para mulheres, ficou abaixo dos valores de referência encontrados no estudo de Nerilo, et al. (2010). Este fato não era o esperado, já que não é recomendado o uso de inseticidas inibidores da colinesterase para a cultura da uva. Uma explicação para o ocorrido seria a exposição ambiental desses viticultores, devido à proximidade das fazendas com outras lavouras que não a uva. Outra seria o uso de mancozebe, um estudo relata que este fungicida, pode apresentar uma fraca atividade anticolinesterásica (Kimura, et al., 2005).

Com relação aos resultados envolvendo a percepção do agricultor familiar quanto as políticas sociais, foi difícil encontrar pesquisas que abordassem esta questão, portanto houve ineficácia na discussão deste tópico. Um dos raros estudos encontrado na literatura brasileira foi de Scardoelli, et al. (2011) que concluiu, em sua pesquisa realizada nesta mesma região, que a alta incidência de intoxicações envolvendo o trabalho com agrotóxicos merece atenção especial, desenvolvimento de estratégias preventivas e planejamento de ações direcionadas para o trabalhador rural.

Os resultados apresentados demonstraram que grande parte desta população depende do SUS, não tem assistência por parte da saúde pública do município, e relataram que a saúde é o serviço público em que a população tem o pior atendimento. Fato que agrava a situação em que a intoxicação crônica não é identificada e muito menos notificada.

O estudo de Buralli, et al. (2018) envolvendo agricultores do estado do Rio de Janeiro, também relatou falhas nos serviços oferecidos por parte da saúde pública. De acordo com o autor, a lei brasileira determina que os trabalhadores rurais devem passar por exames e consultas médicas periódicas, porém na realidade isso não acontece. Os resultados obtidos neste trabalho reforçam que os agricultores não recebem o monitoramento de saúde adequado por parte do poder público.

\section{Considerações Finais}

Apesar das limitações de um estudo de caso municipal e da amostragem intencional, esta pesquisa apresenta pontos fortes como: (a) evidência de sintomas relacionados à exposição crônica aos fungicidas entre os participantes do estudo, e ausência de monitoramento com periodicidade pela saúde pública; (b) foi utilizado um documento validado para a elaboração dos instrumentos de coleta de dados e discussão dos resultados (c) o município de estudo é caracterizado por produção familiar; (d) este trabalho apresenta a percepção dos trabalhadores rurais quanto ao serviço público oferecido pelo município (e) os dados referentes à percepção dos trabalhadores acerca das políticas sociais e saúde, foram apresentados para autoridades da prefeitura do município.

Os resultados obtidos com esta pesquisa reforçam a importância do monitoramento ocupacional periódico dos agricultores, visto que essa população se expõe aos agrotóxicos sem a proteção adequada por muito anos, e apresenta sinais e sintomas que podem estar relacionados a esta exposição.

A falta do uso de EPI por trabalhadores rurais ocorre em outras partes do país e do mundo, portanto, somente a orientação quanto ao perigo não é suficiente para conscientizar esta população a fazerem uso dos equipamentos de proteção. $\mathrm{O}$ ideal é seguir para práticas de redução do uso dos agrotóxicos, como o IDR-Paraná de Marialva faz com esta população de viticultores, associando medidas alternativas e diminuindo o uso dos agrotóxicos para assim reduzir a exposição.

Vale ressaltar a importância de novos estudos com esta população e também com outras populações envolvendo a agricultura familiar, já que outros estudos relataram falhas em serviços da área de saúde pública. Sugerimos ações de capacitação às equipes de saúde da atenção primária, que podem ser programadas em cooperação com o município a fim de reduzir os riscos e danos à saúde dos trabalhadores rurais.

\section{Agradecimentos}


Este trabalho contou com o apoio da Coordenação de Aperfeiçoamento de Pessoal de Nível Superior (CAPES, Brasília, Brasil); Secretaria de Estado de Ciência, Tecnologia e Ensino Superior (SETI, Curitiba, Paraná, Brasil); Unidade de Gestão do Fundo do Paraná (SETI/UGF, Curitiba, Paraná, Brasil) número do protocolo [35591.433.38503.22082016] Programa de Extensão "Universidade Sem Fronteiras" (USF) e Instituto de Desenvolvimento Rural do Paraná (IDR-PARANÁ, Marialva, Paraná, Brasil).

\section{Referências}

Agência de Defesa Agropecuária do Paraná (2019a). Agrotóxicos no Paraná. http://celepar07web.pr.gov.br/agrotoxicos/pesquisar.asp Agência de Defesa Agropecuária do Paraná (2019b). Agrotóxicos- Outros. Dormex. http://www.adapar.pr.gov.br/arquivos/ File/defis/DFI/Bulas/Outros/dormex160218.pdf

Agência Nacional de Vigilância Sanitária (2015). Consulta Pública $\mathrm{n}^{\circ}$ 87, de 2 de outubro de 2015. http://inmetro.gov.br/barreirastecnic as/pontofocal/textos/regulamentos/BRA_648.pdf

Alencar, J. A. (2010). Sistema de Produção - Cultivo da Videira. Normas gerais sobre o uso de agrotóxicos: Embrapa.

Brasil. (1989). Lei $\mathrm{n}^{\mathrm{o}}$ 7.802, de 11 de julho de 1989. Dispõe sobre a pesquisa, a experimentação, a produção, a embalagem e rotulagem, o transporte, o armazenamento, a comercialização, a propaganda comercial, a utilização, a importação, a exportação, o destino final dos resíduos e embalagens, o registro, a classificação, o controle, a inspeção e a fiscalização de agrotóxicos, seus componentes e afins, e dá outras providências. http://www.planalto.gov.br/ccivil_03/leis/L7802.htm.

Brasil. (2016). Decreto n ${ }^{\circ}$ 8.948, de 29 de dezembro de 2016. Que dispõe sobre o valor do salário mínimo e a sua política de valorização de longo prazo. Diário Oficial da União.

Buralli, R. J., Ribeiro, H., Mauad, T., Amato-Lourenço, L. F., Salge, J. M., Diaz-Quijano, F. A., Leão, R. S., Marques, R. C., Silva, D. S., \& Guimarães, J. R. D. (2018). Respiratory Condition of Family Farmers Exposed to Pesticides in the State of Rio de Janeiro, Brazil. Int J Environ Res Public Health, 15(6). https://doi.org/10.3390/ijerph15061203

Carneiro, F. F. (2015). Dossiê ABRASCO: um alerta sobre os impactos dos agrotóxicos na saúde: EPSJV/Expressão Popular.

Departamento de Informática do Sistema Único de Saúde. (2019). Intoxicação Exógena - Notificações registradas no SINAN NET - Brasil. http://tabnet.datasus.gov.br/cgi/tabcgi.exe?sinannet/cnv/Intoxbr.def

Ellman, G. L., Courtney, K. D., Andres, V., Jr., \& Feather-Stone, R. M. (1961). A new and rapid colorimetric determination of acetylcholinesterase activity. Biochem Pharmacol, 7, 88-95. https://doi.org/10.1016/0006-2952(61)90145-9

Furlong, M., Tanner, C. M., Goldman, S. M., Bhudhikanok, G. S., Blair, A., Chade, A., Comyns, K., Hoppin, J. A., Kasten, M., Korell, M., Langston, J. W., Marras, C., Meng, C., Richards, M., Ross, G. W., Umbach, D. M., Sandler, D. P., \& Kamel, F. (2015). Protective glove use and hygiene habits modify the associations of specific pesticides with Parkinson's disease. Environ Int, 75, 144-150. https://doi.org/10.1016/j.envint.2014.11.002

García-García, C. R., Parrón, T., Requena, M., Alarcón, R., Tsatsakis, A. M., \& Hernández, A. F. (2016). Occupational pesticide exposure and adverse health effects at the clinical, hematological and biochemical level. Life Sci, 145, 274-283. https://doi.org/10.1016/j.1fs.2015.10.013

Gunnarsson, L. G., \& Bodin, L. (2019). Occupational Exposures and Neurodegenerative Diseases-A Systematic Literature Review and Meta-Analyses. Int J Environ Res Public Health, 16(3). https://doi.org/10.3390/ijerph16030337

Harlin, K. S., \& Ross, P. F. (1990). Enzymatic-spectrophotometric method for determination of cholinesterase activity in whole blood: collaborative study. $J$ Assoc Off Anal Chem, 73(4), 616-619.

Hungaro, A. A., Correia, L. M., Santos Silvino, M. C., Moura Rocha, S., Ferreira Martins, B., \& de Oliveira, M. L. F. (2015). Pesticide poisoning: records of a toxicological assistance sentinel service. Ciencia, Cuidado e Saude, 14(3).

Instituto Brasileiro do Meio Ambiente e dos Recursos Naturais Renováveis (2018). Relatórios de comercialização de agrotóxicos/ Boletins anuais/ Boletim de 2018. https://www.ibama.gov.br/agrotoxicos/relatorios-de-comercializacao-de-agrotoxicos

Kim, K. H., Kabir, E., \& Jahan, S. A. (2017). Exposure to pesticides and the associated human health effects. Sci Total Environ, 575, 525-535. https://doi.org/10.1016/j.scitotenv.2016.09.009

Kimura, K., Yokoyama, K., Sato, H., Nordin, R. B., Naing, L., Kimura, S., Okabe, S., Maeno, T., Kobayashi, Y., Kitamura, F., \& Araki, S. (2005). Effects of pesticides on the peripheral and central nervous system in tobacco farmers in Malaysia: studies on peripheral nerve conduction, brain-evoked potentials and computerized posturography. Ind Health, 43(2), 285-294. https://doi.org/10.2486/indhealth.43.285

Lopes, C. V. A., \& de Albuquerque, G. S. C. (2018). Agrochemicals and their impacts on human and environmental health: a systematic review. Saúde em Debate, 42, 518-534.

Medda, E., Santini, F., De Angelis, S., Franzellin, F., Fiumalbi, C., Perico, A., Gilardi, E., Mechi, M. T., Marsili, A., Citroni, A., Leandri, A., Mantovani, A., Vitti, P., \& Olivieri, A. (2017). Iodine nutritional status and thyroid effects of exposure to ethylenebisdithiocarbamates. Environ Res, 154, 152-159. https://doi.org/10.1016/j.envres.2016.12.019 
Ministério do Trabalho e Emprego (1978). Norma regulamentadora NR 7 - Programa de controle médico de saúde ocupacional. Publicada na Portaria SEPRT n. ${ }^{\circ} 6.734$, de 10 de março de 2020. https://enit.trabalho.gov.br/portal/images/Arquivos_SST/SST_NR/NR-07.pdf.

Mossini, S. A. G.; Nishiyama, P. \& Machado, P. A. L. (2017). Agrotóxicos - aspectos gerais e implicações para a saúde humana. Em: Villalobos JUG, Fazolli SA, editors. Agrotóxicos. um enfoque multidisciplinar. EDUEM p. 63-83.

Mostafalou, S., \& Abdollahi, M. (2013). Pesticides and human chronic diseases: evidences, mechanisms, and perspectives. Toxicol Appl Pharmacol, 268(2), 157-177. https://doi.org/10.1016/j.taap.2013.01.025

Nascimento, S., Göethel, G., Gauer, B., Sauer, E., Nardi, J., Cestonaro, L., Correia, D., Peruzzi, C., Mota, L., Machry, R. V., Furlanetto, T. W., Saint' Pierre, T., Gioda, A., Arbo, M. D., \& Garcia, S. C. (2018). Exposure to environment chemicals and its possible role in endocrine disruption of children from a rural area. Environ Res, 167, 488-498. https://doi.org/10.1016/j.envres.2018.07.039

Nerilo, S. B. (2010). Atividade da acetilcolinesterase e butirilcolinesterase de indivíduos expostos e não expostos a inseticidas inibidores da colinesterase (Dissertação). Universidade Estadual de Maringá.

Pereira, A. S., Shitsuka, D. M., Parreira, F. J. \& Shitsuka, R. (2018). Metodologia da Pesquisa Científica. UFSM.

Raksanam, B., Taneepanichskul, S., Robson, M. G., \& Siriwong, W. (2014). Health risk behaviors associated with agrochemical exposure among rice farmers in a rural community, Thailand: a community-based ethnography. Asia Pac J Public Health, 26(6), 588-595. https://doi.org/10.1177/1010539512466426

Rocha, G. H., Lini, R. S., Barbosa, F., Jr., Batista, B. L., de Oliveira Souza, V. C., Nerilo, S. B., Bando, E., Mossini, S. A. G., \& Nishiyama, P. (2015). Exposure to heavy metals due to pesticide use by vineyard farmers. Int Arch Occup Environ Health, 88(7), 875-880. https://doi.org/10.1007/s00420-014-1010-1

Salgado, P. E. T.; Larini, L. \& Lepera, J. S. (1987) Metais. Em: Larini L. Toxicologia. Segunda edição. Manole p. 114-135.

Scardoelli, M. G. C., Buriola, A. A., de Oliveira, M. L. F., \& Waidman, M. A. P. (2011). Intoxicações por agrotóxicos notific adas na $11^{a}$ regional de saúde do estado do Paraná. Ciência, Cuidado e Saúde, 10(3), 549-555.

Secretaria de Estado da Saúde do Paraná, Superintendência de Vigilância em Saúde, Centro Estadual de Saúde do Trabalhador. (2013). Protocolo de avaliação das intoxicações crônicas por agrotóxico crônicas por agrotóxico. Curitiba.

Soares, W. L., \& Porto, M. F. (2012). Pesticide use and economic impacts on health. Rev Saude Publica, 46(2), 209-217. https://doi.org/10.1590/s003489102012005000006

Sousa, J. A., Feitosa, H. O., Carvalho, C. M., Pereira, C. F., Feitosa, S. O., \& Silva, S. L. (2016). Percepção dos produtores rurais quanto ao uso de agrotóxicos Revista Brasileira de Agricultura Irrigada, 10(5), 976-989.

Van der Mark, M., Brouwer, M., Kromhout, H., Nijssen, P., Huss, A., \& Vermeulen, R. (2012). Is pesticide use related to Parkinson disease? Some clues to heterogeneity in study results. Environ Health Perspect, 120(3), 340-347. https://doi.org/10.1289/ehp.1103881

Viana, L. S., Queiroz, I. F. R., Ribeiro, M. A., Albuquerque, I. M. A. N., \& Neto, F. R. G. X. (2017). Contextualizando a realidade do uso de agrotóxicos na agricultura familiar. Extensão em Ação, 1(13), 54-68.

Yan, D., Zhang, Y., Liu, L., Shi, N., \& Yan, H. (2018). Pesticide exposure and risk of Parkinson's disease: Dose-response meta-analysis of observational studies. Regul Toxicol Pharmacol, 96, 57-63. https://doi.org/10.1016/j.yrtph.2018.05.005

Ye, M., Beach, J., Martin, J. W., \& Senthilselvan, A. (2017). Pesticide exposures and respiratory health in general populations. J Environ Sci (China), 51, 361370. https://doi.org/10.1016/j.jes.2016.11.012

Zeneratti, F. L. (2019). Cooperativismo e recriação camponesa: contribuições e limites dos modelos de cooperativismo empresarialista e camponês na mesorregião norte central paranaense (resumo de tese). Bol. geogr. 37(3), 279-280. 